\title{
MINIMAL CLADE SIZE IN THE BOLTHAUSEN-SZNITMAN COALESCENT
}

\author{
FABIAN FREUND, * University of Hohenheim \\ ARNO SIRI-JÉGOUSSE,** Centro de Investigación en Matemáticas, A.C.
}

\begin{abstract}
In this article we show the asymptotics of distribution and moments of the size $X_{n}$ of the minimal clade of a randomly chosen individual in a Bolthausen-Sznitman $n$-coalescent for $n \rightarrow \infty$. The Bolthausen-Sznitman $n$-coalescent is a Markov process taking states in the set of partitions of $\{1, \ldots, n\}$, where $1, \ldots, n$ are referred to as individuals. The minimal clade of an individual is the equivalence class the individual is in the time of the first coalescence event this individual participates in. We also provide exact formulae for the distribution of $X_{n}$. The main tool used is the connection of the Bolthausen-Sznitman $n$-coalescent with random recursive trees introduced by Goldschmidt and Martin (2005). With it, we show that $X_{n}-1$ is distributed as the size of a uniformly chosen table in a standard Chinese restaurant process with $n-1$ customers.
\end{abstract}

Keywords: Minimal clade size; Bolthausen-Sznitman $n$-coalescent; Chinese restaurant process

2010 Mathematics Subject Classification: Primary 60C05

Secondary 05C80; 60G09; 60F05; 60J27; 92D25

\section{Introduction}

The Bolthausen-Sznitman $n$-coalescent is a time-homogeneous Markov process $\left(\Pi_{t}^{(n)}\right)_{t \geq 0}$ whose state space is the set of partitions of $\{1, \ldots, n\}$. The only possible transitions in this process are those in which several blocks of a partition are merged (or coalesced) into one new block. Only one new block can be formed in a transition (no simultaneous mergers). Each $k$-tuple of $b$ present blocks is merged to a new block at rate $(k-2) !(b-k) ! /(b-1)$ !. The Bolthausen-Sznitman $n$-coalescent is a member of the $\Lambda$ - $n$-coalescent family (which were introduced independently by Sagitov [24] and Pitman [22]). A $\Lambda$ - $n$-coalescent is again a timehomogeneous, continuous-time Markov process whose state space is the set of partitions of $\{1, \ldots, n\}$. The possible transitions are again mergers of multiple blocks into a new block. Each merger of $k$ blocks among $b$ happens with rate

$$
\int_{[0,1]} x^{k-2}(1-x)^{n-k} \Lambda(\mathrm{d} x)
$$

for a finite measure $\Lambda$ on $[0,1]$. Note that the Bolthausen-Sznitman coalescent has $\Lambda=U_{[0,1]}$, the uniform distribution on $[0,1]$. Each $\Lambda$ - $n$-coalescent can be represented as a random tree

Received 20 March 2013; revision received 24 June 2013.

* Postal address: Crop Plant Biodiversity and Breeding Informatics Group (350b), Institute of Plant Breeding, Seed Science and Population Genetics, University of Hohenheim, Fruwirthstrasse 21, 70599 Stuttgart, Germany.

Email address: fabian.freund@uni-hohenheim.de

** Postal address: Centro de Investigación en Matemáticas, Calle Jalisco s/n, Col. Mineral de Valenciana, 36240 Guanajuato, Mexico. Email address: arno@cimat.mx 
with $n$ leaves $\{1, \ldots, n\}$ and random branch lengths by representing each merger as an internal node in the tree (the branch lengths are given by the waiting times for the mergers, time is measured starting from the leaves). Also, note that a $\Lambda$ - $n$-coalescent at time $t$ forms a random exchangeable partition of $\{1, \ldots, n\}$.

The Bolthausen-Sznitman $n$-coalescent was introduced by Bolthausen and Sznitman in 1998 (see [5]). It has connections to population genetics and physics. In mathematical physics, it appears in the context of spin glasses (see [5] and [6]). It also seems to be a suitable model for the genealogy of a sample of $n$ alleles/genes/haplotypes in several models for selection in population genetics (see [2], [8], [9], [12], and [21]; see also the survey [7]). Note that this is in contrast to the standard model for a genealogical tree of a sample of $n$ alleles/genes/haplotypes which is Kingman's $n$-coalescent $\left(\Lambda=\delta_{0}\right.$, only two blocks merge at a time, introduced in [20]). Also, note that, due to the interpretation of the Bolthausen-Sznitman $n$-coalescent as a genealogical tree, we refer to $\{1, \ldots, n\}$ as individuals.

Here, we focus on the Bolthausen-Sznitman $n$-coalescent as a model for a genealogical tree which depicts the ancestry of $n$ alleles sampled at a genetic locus. Since the genealogical tree is often endowed with a mutation structure which is interpreted under the infinitely-many sites model, we assume a locus consisting of many nucleotide sites, for example, a gene. Different alleles can thus also be seen as different haplotypes spanning over the nucleotide sites of this locus. One piece of important information coded in the genealogy is the relatedness of an allele randomly chosen from the sample to the rest of the sample. There are two functionals/statistics of the genealogical tree which transport complementary information about this relatedness. The first functional is the length $E_{n}$ of an external branch chosen at random from the $n$ external branches associated with the leaves $\{1, \ldots, n\}$ of the tree, introduced by Fu and $\mathrm{Li}$ [15]. Here $E_{n}$ gives the time that the chosen allele has to evolve independently of the rest of the sample (e.g. by mutation). This gives a measure of the genetic uniqueness of this allele relative to the rest of the sample. The second functional is the size $X_{n}$ of the minimal clade containing the randomly chosen allele, introduced by Blum and François [4]. The minimal clade can be defined in different, yet equivalent ways. The minimal clade is

- the equivalence class that contains the (randomly chosen) allele $i \in\{1, \ldots, n\}$ at the first time $i$ is merged,

- all leaves of the subtree rooted at the most recent ancestor of allele $i$,

- all descendants of the most recent ancestor of allele $i$.

The minimal clade can also be seen as the smallest family containing $i$. The size of the minimal clade gives the complementary information of how many individuals share the genealogy with allele $i$ 'after' time $E_{n}$ (note that since we measure time from the leaves to the root, 'after' $E_{n}$ actually means further back in time).

The external branch length has already been well analysed for several $\Lambda$ - $n$-coalescents in the literature. Its distribution follows a recursion and its asymptotics for a sample size $n \rightarrow \infty$ are known for various $\Lambda-n$-coalescents (see [4], [10], [13], [14], and [16]). For the minimal clade size, though, only results for Kingman's $n$-coalescent $\left(\Lambda=\delta_{0}\right.$, only two blocks merge at a time) are known (including asymptotics for $n \rightarrow \infty$; see [4]).

The purpose of this paper is to analyse the distribution of the minimal clade size $X_{n}$ in the case of the Bolthausen-Sznitman $n$-coalescent and its asymptotics for a sample size $n \rightarrow \infty$. We will exploit the construction of the Bolthausen-Sznitman $n$-coalescent using a random recursive tree introduced by Goldschmidt and Martin [17] to prove our results. First, we observe that 
it follows from this construction that the process describing the set of relatives of a randomly chosen individual in the Bolthausen-Sznitman $n$-coalescent process (which is its equivalence class without the individual itself) is equal in law to the time-reversed process describing the set of nonrelatives of the chosen individual (all individuals in different equivalence classes to the chosen individual). This shows that the minimal clade size is actually distributed as the sum $M_{n}$ of the sizes of all blocks not containing 1 which participate in the last collision in the $n$-coalescent. Convergence in distribution of properly scaled $M_{n}$ for $n \rightarrow \infty$ has already been shown already by Goldschmidt and Martin [17] and, thus, the same asymptotic behavior holds for $X_{n}$, namely, $(\log n)^{-1} \log X_{n}$ converges in distribution to the uniform distribution on [0,1].

Note that, owing to the connection between the random recursive tree and the standard Chinese Restaurant process, we observe that $X_{n}-1$ and $M_{n}$ are distributed as the size of a uniformly chosen table (not chosen by a size-biased pick!) in the Chinese restaurant process (again for $M_{n}$ in accordance to [17]). This allows us to give several formulae for the exact distribution of $X_{n}$. Using these, we show that, for $k \in \mathbb{N},\left(\log n / n^{k}\right) \mathbb{E}\left(X_{n}^{k}\right) \rightarrow 1 / k$ for $n \rightarrow \infty$, which gives complementary information to the weak convergence result.

\section{Minimal clade size in the Bolthausen-Sznitman $\boldsymbol{n}$-coalescent}

Set $[n]:=\{1, \ldots, n\}$ and $[n]_{0}:=\{0, \ldots, n\}$. For a partition $\eta$ of $[n]$, let $C_{i}(\eta)$ denote the equivalence class of $i \in[n]$ and let $\left|C_{i}(\eta)\right|$ be its size. Let $\left(\Pi_{t}^{(n)}\right)_{t \geq 0}$ be a $\Lambda-n$-coalescent. Since we want to look at the minimal clade size of a randomly chosen allele in the sample whose genealogy is given by $\left(\Pi_{t}^{(n)}\right)_{t \geq 0}$, define $I$ as a uniform pick from $[n]$ independent of the $n$-coalescent. Now, first define the length of a randomly chosen external branch (associated with the randomly chosen $I \in[n])$ by

$$
E_{n}:=\inf \left\{t \geq 0, C_{I}\left(\Pi_{t}^{(n)}\right) \neq\{I\}\right\} .
$$

Now we define the size of the minimal clade of the randomly chosen allele $I$ as

$$
X_{n}:=\left|C_{I}\left(\Pi_{E_{n}}^{(n)}\right)\right| .
$$

Note that, owing to exchangeability, we do not change the distributions of $E_{n}$ and $X_{n}$ if we assume $I=1$. Also, note that, owing to the interpretation of an $n$-coalescent as a genealogical tree, we refer to $\{1, \ldots, n\}$ as individuals.

From now on, we will abbreviate the size of the minimal clade of $I=1$ with the minimal clade size.

The minimal clade of individual 1 is the size of the equivalence class of 1 at the first coalescence event that the individual participates in. Goldschmidt and Martin [17] analysed the behavior of the total mass $M_{n}$ of the equivalence classes not containing 1 at the last coalescence event in the Bolthausen-Sznitman $n$-coalescent (see [17, Theorem 3.1]). Note that $M_{n}$ can also be written as $n-\left|C_{1}\left(\Pi_{\tau_{n}-}^{(n)}\right)\right|$, where $\tau_{n}$ is the waiting time for the last coalescence event. Both $X_{n}$ and $M_{n}$ are functionals of the equivalence class of 1 in the Bolthausen-Sznitman $n$-coalescent at different times. Thus, it is interesting how the equivalence class of 1 changes over time. It will only grow by merging with other equivalence classes at coalescence times, but not necessarily at all coalescence times. We define $S_{i}^{(n)}$ as the equivalence class of 1 after the $i$ th merging event which 1 participates in. What are the properties of $\left(S_{i}^{(n)}\right)_{i \in\left[\kappa_{n}\right]_{0}}$, where $\kappa_{n}$ is the number of merging events 1 participates in? The results from [17] answer this question. There, the authors showed a construction of the Bolthausen-Sznitman $n$-coalescent by applying 
a cutting procedure to a random recursive tree and used it, among answering other questions, to analyse $M_{n}$.

We will show in detail that this construction enables us to analyse the behavior of $S^{(n)}$ and that $S^{(n)}$ can be expressed in terms of a Chinese restaurant process. Note that this is just the line of reasoning from [17]. Let us quickly recall the construction of the Bolthausen-Sznitman $n$-coalescent from a random recursive tree from [17, Proposition 2.2] as well as the connection to the Chinese restaurant process. Here, we give a simplified version just constructing the jump chain of the $n$-coalescent.

We start with a random recursive tree with $n$ vertices, i.e. a uniformly distributed random variable on the set of all recursive trees with $n$ vertices $1, \ldots, n$ rooted in 1 (here the branches carry no length information). Now construct the jump chain as follows.

- Choose an edge at random.

- Cut the tree at this edge. All labels that are in the subtree not containing the root are added to the node of the subtree containing the root which was adjacent to the edge cut.

- Define a partition by taking the labels at each node of the subtree containing the root. This partition has the same law as the Bolthausen-Sznitman $n$-coalescent after the first jump.

- Repeat steps 1-3 with the subtree containing the root. This leads to partitions which have the same law as the Bolthausen-Sznitman $n$-coalescent after the 2nd, 3rd, etc. jump.

Now, we present the connection of the random recursive tree with the Chinese restaurant process.

First, recall that the standard Chinese restaurant process is a sequential construction of a uniform permutation of $[n]$. Imagine a restaurant with tables $1,2,3, \ldots$ with infinitely many chairs. Customers $1, \ldots, n$ sit down at the tables according to the following rule.

- Customer 1 sits at table 1 .

- If $i-1$ customers have taken their seat, the $i$ th customer sits with equal probability at one of the following $i$ places:

- on a chair directly to the left of an already seated customer (possibly between customers),

- at a previously unoccupied table.

Writing down the customers at each table in seating order, we get the cycles of a uniform random permutation of $[n]$. If we only record the customers at each table, but not the seating order, we get an exchangeable partition of $[n]$ whose distribution is given by the celebrated Ewens sampling formula with mutation parameter $\theta=1$ (see, e.g. [1, Equation (1.3)]). More information on this process can be found in [23, Chapter 3.1]. We will abbreviate a standard Chinese restaurant process with $n$ customers by $\operatorname{CRP}(n)$.

$\operatorname{A~CRP}(n-1)$ can be found in a random recursive tree with $n$ vertices in the following way (see [17, pp. 724-725]). We define a subtree of ' 1 ' in the random recursive tree as a rooted subtree whose root is adjacent (connected by one edge) to the root ' 1 ' of the whole tree. Then the subtrees of ' 1 ' form a exchangeable partition of $\{2, \ldots, n\}$ which can be described as a $\operatorname{CRP}(n-1)$ with customers labeled $2, \ldots, n$. The following lemma is just a write-up of the line of reasoning from [17, p. 725] and gives a discrete analog of a part of [22, Corollary 16] (in [17], the line of reasoning presented here is a part of an alternative proof for [22, Corollary 16]). 
Lemma 1. (Practically from [17].) Let $\kappa_{n}$ be the number of collisions in a Bolthausen-Sznitman $n$-coalescent individual 1 participates in. For $i \in\left[\kappa_{n}\right]_{0}$, let $S_{i}^{(n)}$ be the equivalence class of 1 in the Bolthausen-Sznitman n-coalescent after the ith collision (involving 1$)$. For a $\operatorname{CRP}(n-1)$ with $K_{n-1}$ tables and with customers labelled $\{2, \ldots, n\}$, let $R T_{1}, \ldots, R T_{K_{n-1}}$ be the tables in random order. Then $S^{(n)}=\left(S_{i}^{(n)}\right)_{i \in\left[\kappa_{n}\right]_{0}}$ is distributed as $\left(\{1\} \cup \bigcup_{j \in[i]} R T_{j}\right)_{i \in\left[K_{n-1}\right]}$.

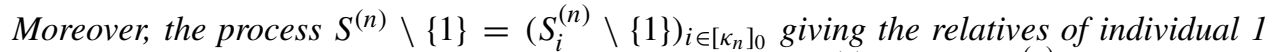
through time is distributed as the time-reversed process $[n] \backslash S^{(n)}=\left([n] \backslash S_{\kappa_{n}-i}^{(n)}\right)_{i \in\left[\kappa_{n}\right]_{0}}$ giving the nonrelatives of individual 1.

If the Bolthausen-Sznitman $n$-coalescent is constructed via cutting a random recursive tree, this lemma can be described more graphically: The equivalence class of 1 grows by adding tables chosen uniformly at random from the Chinese restaurant process with $n-1$ customers given by the subtrees of ' 1 ' in the random recursive tree.

Note that we actually choose tables at random and not individuals sitting at tables, so we do not make size-biased picks.

Proof of Lemma 1. We construct the Bolthausen-Sznitman $n$-coalescent via cutting a random recursive tree. The equivalence class of 1 is merged with other equivalence classes as soon as an edge adjacent to the root is cut in the random recursive tree. The equivalence class of 1 is then merged with the subtree of ' 1 ' which is connected by that edge. Since the edges are chosen at random, this means that a uniformly chosen table of the $\operatorname{CRP}(n-1)$ given by the subtrees of ' 1 ' is merged with the class of 1 .

Since $X_{n}-1=\left|S_{1}^{(n)} \backslash\{1\}\right|$ and $M_{n}=\left|[n] \backslash S_{\kappa_{n}-1}^{(n)}\right|$, Lemma 1 shows that $X_{n}-1$ and $M_{n}$ have the same distribution, namely that both are distributed as the size of a uniformly chosen table in a $\operatorname{CRP}(n-1)$. This means that the known results for the asymptotics of $M_{n}$ which are given in [17, Theorem 3.1] are valid for $X_{n}-1$ and, owing to a Slutsky argument, are also valid for $X_{n}$.

Theorem 1. Let $n \in\{2,3, \ldots\}$. Let $X_{n}$ be the minimal clade size in the Bolthausen-Sznitman $n$-coalescent. Then $X_{n}-1$ is distributed as the size of a randomly chosen table in a $\operatorname{CRP}(n-1)$ and

$$
\frac{\log X_{n}}{\log n} \rightarrow U_{[0,1]}
$$

holds in distribution for $n \rightarrow \infty$, where $U_{[0,1]}$ is the uniform distribution on $[0,1]$.

Additionally to this result, we give the complementary information of the exact law of $X_{n}$ and of the first-order behavior of all moments of $X_{n}$ for $n \rightarrow \infty$.

Theorem 1 states that the distribution of $X_{n}$ can be expressed in terms of the Chinese restaurant process. We will use this to derive three formulae for the distribution of $X_{n}$. Let us recall two possibilities to look at the distribution of customers at tables in a $\operatorname{CRP}(n)$. Recall that this distribution in a $\operatorname{CRP}(n)$ is given by the Ewens sampling formula with mutation parameter $\theta=1$. We use two different possibilities to look at the Ewens sampling formula in (2) and (3) below. First, we can record how many tables in a $\operatorname{CRP}(n)$ have exactly $i$ customers, which we denote by $A_{i}^{(n)}$ for each $i \in[n]$. Then, for $a_{1}, \ldots, a_{n} \in[n]_{0}$ with $\sum_{i \in[n]} i a_{i}=n$, we have

$$
\mathbb{P}\left(A_{1}^{(n)}=a_{1}, \ldots, A_{n}^{(n)}=a_{n}\right)=\prod_{i=1}^{n} \frac{1}{a_{i} ! i^{a_{i}}} .
$$


On the other hand, we can record the probability that certain sets of customers sit at tables $1,2, \ldots$ (this forms a partition $\eta$ of $[n]$ ). The probability that we find a certain partition $\eta$ (with blocks ordered by their least element) of $[n]$ with $k$ occupied tables and $n_{i}$ customers at the $i$ th occupied table is

$$
\mathbb{P}(\operatorname{CRP}(n)=\eta)=\frac{1}{n !} \prod_{i \in[k]}\left(n_{i}-1\right) ! .
$$

This leads to several possibilities to express the distribution of $X_{n}$.

Lemma 2. Let $n \in\{2,3, \ldots\}$. Let $X_{n}$ be the minimal clade size in a Bolthausen-Sznitman $n$-coalescent. For $m \in \mathbb{N}$, let $A_{i}^{(m)}$ be the number of tables with exactly $i$ customers in a $\operatorname{CRP}(m)$, and let $K_{m}=\sum_{i \in[n-1]} A_{i}^{(m)}$ be the number of occupied tables. Define $K_{0}=0$ ('empty restaurant'). Then, for $j \in[n-1]$, the following statements hold.

(a) Defining $\Gamma_{n}=\left\{a_{1}, \ldots, a_{n-1} \in[n-1]_{0}, \sum_{i=1}^{n-1} i a_{i}=n-1\right\}$,

$$
\mathbb{P}\left(X_{n}=j+1\right)=\mathbb{E}\left(\frac{A_{j}^{(n-1)}}{K_{n-1}}\right)=\sum_{\Gamma_{n}} \frac{a_{j}}{\sum_{i=1}^{n-1} a_{i}} \prod_{i=1}^{n-1} \frac{1}{a_{i} ! i^{a_{i}}} .
$$

(b) Defining $\Delta(n, k)=\left\{n_{1}, \ldots, n_{k} \in[n], \sum_{i=1}^{k} n_{i}=n\right\}$ for $k \leq n$,

$$
\mathbb{P}\left(X_{n}=j+1\right)=\frac{1}{j} \sum_{k=1}^{n-1-j} \frac{1}{(k+1) !} \sum_{\Delta(n-1-j, k)} \frac{1}{n_{1} \cdots n_{k}}
$$

for $j<n-1$ and $\mathbb{P}\left(X_{n}=n\right)=1 /(n-1)$.

(c) Let $B_{1}, B_{2}, \ldots$ be independent Bernoulli-distributed random variables with success probability $1 / i$ for $B_{i}$. Then

$$
\mathbb{P}\left(X_{n}=j+1\right)=\frac{1}{j} \mathbb{E}\left(\frac{1}{1+K_{n-1-j}}\right)=\frac{1}{j} \mathbb{E}\left(\frac{1}{1+\sum_{i=1}^{n-1-j} B_{i}}\right) .
$$

Note that the above lemma also holds for $M_{n}$ and the size $R T_{n-1}$ of a randomly chosen table in a $\operatorname{CRP}(n-1)$ (just replace $j+1$ with $j$ ). Also, note that this result provides a very rare example where an exact law is obtained for a functional of an exchangeable non-Kingman, non-starshaped $n$-coalescent.

Proof of Lemma 2. Owing to Theorem 1, we know that $X_{n}-1$ is distributed as the size of a randomly chosen table in a $\operatorname{CRP}(n-1)$. Given the table counts $A_{1}^{(n-1)}, \ldots, A_{n-1}^{(n-1)}$, the probability that we randomly choose a table with $j$ customers is

$$
\frac{A_{j}^{(n-1)}}{\sum_{i=1}^{n-1} A_{i}^{n-1}}=\frac{A_{j}^{(n-1)}}{K_{n-1}}
$$

Summing over the distribution of the table counts given by (2) gives (a).

Now look at the partition $\eta$ of $[n]$ constructed via a $\operatorname{CRP}(n-1)$ whose distribution is given by (3). We are interested in the partition not in the order of the least elements, but in the exchangeable order (meaning that if the partition has $k$ blocks, we order them randomly). 
Let $N_{1}^{(n-1)}, \ldots, N_{k}^{(n-1)}$ be the table sizes in exchangeable order. By combinatorial arguments (see [23, Equation (2.7)]), we obtain

$$
\begin{aligned}
\mathbb{P}\left(N_{1}^{(n-1)}=n_{1}, \ldots, N_{k}^{(n-1)}=n_{k}\right) & =\left(\begin{array}{c}
n-1 \\
n_{1}, \ldots, n_{k}
\end{array}\right) \frac{1}{k !} \underbrace{\frac{1}{(n-1) !} \prod_{i=1}^{k}\left(n_{i}-1\right) !}_{\text {probability of } \eta \text {, least elements }} \\
& =\frac{1}{k ! \prod_{i=1}^{k} n_{i}} .
\end{aligned}
$$

The size of a randomly picked table in the CRP is distributed as $N_{1}^{(n-1)}$. This is just a marginal distribution from the above formula, namely,

$$
\mathbb{P}\left(X_{n}=j+1\right)=\mathbb{P}\left(N_{1}^{(n-1)}=j\right)=\sum_{k=1}^{n-1} \frac{1}{k !} \sum_{\substack{n_{2}, \ldots, n_{k} \in[n-1] \\ j+\sum_{i=2}^{n} n_{i}=n-1}} \frac{1}{j \cdot n_{2} \cdots n_{k}} .
$$

If $j=n-1$, (5) equals $1 /(n-1)$. For $1 \leq j<n-1$, we have

$$
\begin{aligned}
\mathbb{P}\left(X_{n}=j+1\right) & =\sum_{k=2}^{n-j} \frac{1}{k ! j} \sum_{\substack{n_{2}, \ldots, n_{k} \in[n-1-j] \\
\sum_{i=2}^{k} n_{i}=n-1-j}} \frac{1}{n_{2} \cdots n_{k}} \\
& =\frac{1}{j} \sum_{k=1}^{n-1-j} \frac{1}{(k+1) !} \sum_{\Delta(n-1-j, k)} \frac{1}{n_{1} \cdots n_{k}},
\end{aligned}
$$

where the last equation is due to an index shift. This shows (b).

To show (c), we compare (5) with $\mathbb{E}\left(\left(1+K_{n-1-j}\right)^{-1}\right)$. First note that, for $j=n-1$, we have $K_{0}=0$ and, thus,

$$
\frac{1}{n-1} \mathbb{E}\left(\frac{1}{K_{0}+1}\right)=\frac{1}{n-1},
$$

which matches the expression in (b). Now assume that $1 \leq j<n-1$. If we look at the table sizes in exchangeable order, we can compute $\mathbb{P}\left(K_{n-1-j}=k\right)$ by summing the probabilities of all possible configurations of table sizes of exactly $k$ occupied tables in a $\operatorname{CRP}(n-1-j)$. Using (4), this leads to

$$
\begin{aligned}
\mathbb{E}\left(\frac{1}{1+K_{n-1-j}}\right) & =\sum_{k=1}^{n-1-j} \frac{1}{k+1} \sum_{\Delta(n-1-j, k)} \frac{1}{k ! n_{1} \cdots n_{k}} \\
& =\sum_{k=1}^{n-1-j} \frac{1}{(k+1) !} \sum_{\Delta(n-1-j, k)} \frac{1}{n_{1} \cdots n_{k}} .
\end{aligned}
$$

Comparison with (5) yields

$$
\mathbb{P}\left(X_{n}=j+1\right)=\frac{1}{j} \mathbb{E}\left(\frac{1}{1+K_{n-1-j}}\right) .
$$

Recall that $K_{n-1-j}$ is distributed as the number of cycles in a uniform permutation of $[n-1-j]$. It is well known that the number of cycles is distributed as the sum of independent Bernoulli variables $B_{1}, \ldots, B_{n-1-j}$ with success probability $1 / i$ for $B_{i}$ (see, e.g. [1, p. 10]). This proves (c). 
Remark 1. Let $K_{n}$ be the number of occupied tables in a CRP $(n)$. Using $K_{n} \stackrel{\mathrm{D}}{=} \sum_{i \in[n]} B_{i}$ for independent Bernoulli variables $B_{i}$ with success probability $1 / i$, we deduce the recursion

$$
\mathbb{E}\left(\frac{1}{m+K_{n}}\right)=\left(1-\frac{1}{n}\right) \mathbb{E}\left(\frac{1}{m+K_{n-1}}\right)+\frac{1}{n} \mathbb{E}\left(\frac{1}{m+1+K_{n-1}}\right)
$$

for all $m \in \mathbb{N}_{0}$. This recursion gives an efficient method to compute the distribution of the minimal clade size $X_{n}$ by using the representation in Lemma 2(c).

Remark 2. Goldschmidt and Martin [17] proved the weak convergence result for $M_{n}$ for $n \rightarrow$ $\infty$ by using the construction of the Bolthausen-Sznitman $n$-coalescent via cutting a random recursive tree and embedding the random recursive tree in a Yule process. However, as also hinted at by Goldschmidt and Martin (see [17, Corollary 3.3, Remark a)]), the representation of $M_{n}$ as a uniformly chosen table in a $\operatorname{CRP}(n-1)$ allows us to use results about uniform random permutations to prove the convergence part of Theorem 1 without using the Yule process embedding.

Alternative proof of Theorem 1. First, let us look at the distribution function of $\log \left(X_{n}-\right.$ 1) $/ \log (n-1)$. Let $x \in[0,1]$. Using Lemma 2(a), we obtain

$$
\begin{aligned}
\mathbb{P}\left(\frac{\log \left(X_{n}-1\right)}{\log (n-1)} \leq x\right) & =\mathbb{P}\left(X_{n}-1 \leq(n-1)^{x}\right) \\
& =\sum_{j=1}^{\left\lfloor(n-1)^{x}\right\rfloor} \mathbb{E}\left(\frac{A_{j}^{(n-1)}}{K_{n-1}}\right) \\
& =\mathbb{E}\left(\frac{\sum_{j=1}^{\left\lfloor(n-1)^{x}\right\rfloor} A_{j}^{(n-1)}}{\sum_{i=1}^{n-1} A_{i}^{(n-1)}}\right),
\end{aligned}
$$

where $A_{i}^{(n-1)}$ is the number of tables with exactly $i$ customers in a $\operatorname{CRP}(n-1)$. The functional central limit theorem of DeLaurentis and Pittel [11] (see also Hansen [18]) states that

$$
\left(\frac{\sum_{j=1}^{\left\lfloor n^{x}\right\rfloor} A_{j}^{(n)}-x \log n}{\sqrt{\log n}}\right)_{x \in[0,1]} \stackrel{\mathrm{D}}{\rightarrow}\left(B_{x}\right)_{x \in[0,1]}
$$

in $D[0,1]$ when $n \rightarrow \infty$, where $B$ is a standard Brownian motion. This implies that

$$
\frac{\sum_{j=1}^{\left\lfloor n^{x}\right\rfloor} A_{j}^{(n)}}{\log n} \stackrel{\mathbb{P}}{\rightarrow} x
$$

for $x \in[0,1]$. We apply this result to both the numerator and denominator on the right-hand side of (6) (inside of $\mathbb{E}(\cdot)$ ) and get

$$
\frac{\sum_{j=1}^{\left\lfloor(n-1)^{x}\right\rfloor} A_{j}^{(n-1)}}{\sum_{i=1}^{n-1} A_{i}^{(n-1)}}=\frac{\sum_{j=1}^{\left\lfloor(n-1)^{x}\right\rfloor} A_{j}^{(n-1)}}{\log (n-1)} \frac{\log (n-1)}{\sum_{i=1}^{n-1} A_{i}^{(n-1)}} \stackrel{\mathbb{P}}{\rightarrow} x
$$

for $n \rightarrow \infty$. Since $0 \leq \sum_{j=1}^{\left\lfloor(n-1)^{x}\right\rfloor} A_{j}^{(n-1)} / \sum_{i=1}^{n-1} A_{i}^{n-1} \leq 1$ for all $x$ and $n$, we have uniform integrability and, hence,

$$
\mathbb{E}\left(\frac{\sum_{j=1}^{\left\lfloor(n-1)^{x}\right\rfloor} A_{j}^{(n-1)}}{\sum_{i=1}^{n-1} A_{i}^{(n-1)}}\right) \rightarrow x \quad \text { for } n \rightarrow \infty
$$


which shows that $\log \left(X_{n}-1\right) / \log (n-1) \stackrel{\mathrm{D}}{\rightarrow} U_{[0,1]}$, where $U_{[0,1]}$ is the uniform distribution on $[0,1]$. With a Slutsky argument we can show that $\log \left(X_{n}\right) / \log n$ behaves in the same way.

For the asymptotics of moments of $X_{n}$ (as well as $M_{n}$ and $R T_{n}$ ), we use the expression for $\mathbb{P}_{X_{n}}$ from Lemma 2(c), namely,

$$
\mathbb{P}\left(X_{n}=j+1\right)=\frac{1}{j} \mathbb{E}\left(\frac{1}{1+K_{n-1-j}}\right),
$$

where $K_{n}$ is the number of occupied tables in a $\operatorname{CRP}(n)$. Note that $K_{n}$ also gives the number of cycles in a uniform permutation of $\{1, \ldots, n\}$. Thus, the distribution of $K_{n}$ is given by

$$
\mathbb{P}\left(K_{n}=k\right)=\frac{S_{n, k}}{n !} \quad \text { for } k \in[n],
$$

where $\left(S_{n, k}\right)_{k \in[n], n \in \mathbb{N}}$ denotes the absolute Stirling numbers of the first kind.

It is well known that (see, e.g. [23, Equation (3.2)])

$$
\frac{K_{n}}{\log n} \rightarrow 1 \text { almost surely }
$$

for $n \rightarrow \infty$. Since we want to use Lemma 2(c), we are more interested in the behavior of $\mathbb{E}\left(\left(1+K_{n}\right)^{-1}\right)$. From (8), we immediately obtain

$$
\frac{\log n}{1+K_{n}} \rightarrow 1 \text { almost surely }
$$

for $n \rightarrow \infty$. We will need a $L^{1}$-version of (9).

Lemma 3. It holds that

$$
\frac{\log n}{1+K_{n}} \rightarrow 1 \text { in } L^{1} \text { for } n \rightarrow \infty .
$$

Proof. The result follows from (9) and the uniform integrability of $\log n /\left(1+K_{n}\right)$, which we now show. Note that since $\log n /\left(1+K_{n}\right) \leq \log n / K_{n}$ for all $n \in \mathbb{N}$, it suffices to show uniform integrability for $\log n / K_{n}$. Let $A>0$, and let $H_{n}$ be a Poisson-distributed random variable with parameter $\log n$. Note that $H_{n} \stackrel{\mathrm{D}}{=} \sum_{i \in\lfloor\log n\rfloor} H_{i}^{(1)}+R_{n}$, where $\left(H_{i}^{(1)}\right)_{i \in \mathbb{N}}$ are independent and identically distributed random variables that are Poisson distributed with parameter 1 , and $R_{n}$ is Poisson distributed with parameter $\log n-\lfloor\log n\rfloor$, independent of $\left(H_{i}^{(1)}\right)_{i \in \mathbb{N}}$. For $A>1$, we have

$$
\begin{aligned}
\int_{\left\{\log n / K_{n} \geq A\right\}}\left|\frac{\log n}{K_{n}}\right| \mathrm{d} \mathbb{P} & =\log n \sum_{k=1}^{\left\lfloor A^{-1} \log n\right\rfloor} \frac{1}{k} \mathbb{P}\left(K_{n}=k\right) \\
& \stackrel{(7)}{=} \log n \sum_{k=1}^{\left\lfloor A^{-1} \log n\right\rfloor} \frac{S_{n, k}}{n ! k} \\
& =\sum_{k=1}^{\left\lfloor A^{-1} \log n\right\rfloor} \frac{(\log n)^{k}}{k !} \mathrm{e}^{-\log n}\left(\frac{1}{\Gamma(1+r)}+O\left(\frac{k}{(\log n)^{2}}\right)\right) \\
& \leq C \mathbb{P}\left(H_{n} \leq \frac{\log n}{A}\right) \\
& \leq C \mathbb{P}\left(\frac{\sum_{i \in\lfloor\log n\rfloor} H_{i}^{(1)}}{\log n} \leq A^{-1}\right) \\
& \rightarrow 0 \quad \text { for } n \rightarrow \infty,
\end{aligned}
$$


where $r=(k-1)(\log n)^{-1}$ and $C$ is a suitable constant. Here, we use the uniform asymptotic expansion of Hwang (see Theorem 2 of [19]) for the absolute Stirling numbers $S_{n, k}$ of the first kind for $1 \leq k \leq A^{-1} \log n$ (we actually use the cruder version from [1, Equation (1.30)]). The convergence to 0 follows from the law of large numbers for $\left(H_{i}^{(1)}\right)_{i \in \mathbb{N}}$.

This computation shows the uniform integrability of $\log n /\left(1+K_{n}\right)$, completing the proof.

Theorem 2. For $n \in\{2,3, \ldots\}$, let $X_{n}$ be the minimal clade size in the Bolthausen-Sznitman $n$-coalescent. For all $k \in \mathbb{N}$, we have

$$
\frac{\log n}{n^{k}} \mathbb{E}\left(X_{n}^{k}\right) \rightarrow \frac{1}{k}
$$

for $n \rightarrow \infty$.

Again, this theorem is also true for $M_{n}$ and $R T_{n}$ instead of $X_{n}$.

Proof of Theorem 2. Using Lemma 2(c), we obtain

$$
\begin{aligned}
\mathbb{E}\left(\left(X_{n}-1\right)^{k}\right) & =\sum_{j=1}^{n-1} j^{k-1} \mathbb{E}\left(\frac{1}{1+K_{n-1-j}}\right) \\
& =\sum_{l=0}^{n-2}(n-1-l)^{k-1} \mathbb{E}\left(\frac{1}{1+K_{l}}\right) \\
& =\sum_{i=0}^{k-1}\left(\begin{array}{c}
k-1 \\
i
\end{array}\right)(n-1)^{k-1-i}(-1)^{i} \sum_{l=0}^{n-2} l^{i} \mathbb{E}\left(\frac{1}{1+K_{l}}\right) .
\end{aligned}
$$

We will now use Karamata's Tauberian theorem for power series (see [3, Corollary 1.7.3]). It states (among other things) that if $a_{l} \sim c l^{\rho-1} \mathcal{L}(l) / \Gamma(\rho)$ for $n \rightarrow \infty$, where $c, \rho>0$ and $\mathcal{L}$ is a slowly varying function, then $\sum_{k \in[n]} a_{k} \sim c n^{\rho} \mathcal{L}(n) / \Gamma(1+\rho)$. We define $a_{l}:=$ $l^{i} E\left(1 /\left(1+K_{l}\right)\right)$. Note that $a_{l} \sim l^{i} / \log l$ for $l \rightarrow \infty$ due to Lemma 3, which enables us to use the Tauberian theorem for $a_{l}$ with $c:=\Gamma(i+1)=i !, \rho=i+1$, and $\mathcal{L}(n)=(\log n)^{-1}$. For each $i \in[k-1]_{0}$, we thus have

$$
\sum_{l=0}^{n-2} l^{i} \mathbb{E}\left(\frac{1}{1+K_{l}}\right) \sim \frac{1}{i+1} \frac{n^{i+1}}{\log n}
$$

for $n \rightarrow \infty$. This shows that

$$
\begin{aligned}
\frac{\log n}{n^{k}} \mathbb{E}\left(\left(X_{n}-1\right)^{k}\right) & =\sum_{i=0}^{k-1}\left(\begin{array}{c}
k-1 \\
i
\end{array}\right)(n-1)^{k-1-i} \frac{\log n}{n^{k}}(-1)^{i} \sum_{l=0}^{n-2} l^{i} \mathbb{E}\left(\frac{1}{1+K_{l}}\right) \\
& \sim \sum_{i=0}^{k-1}\left(\begin{array}{c}
k-1 \\
i
\end{array}\right) \frac{\log n}{n^{i+1}}(-1)^{i} \frac{1}{i+1} \frac{n^{i+1}}{\log n} \\
& =\sum_{i=0}^{k-1}\left(\begin{array}{c}
k-1 \\
i
\end{array}\right) \frac{(-1)^{i}}{i+1} \\
& =\frac{1}{k} \quad \text { for } n \rightarrow \infty,
\end{aligned}
$$


where the last equation follows by elementary calculations. Thus, for each $k \in \mathbb{N}$, we have established that

$$
\frac{\log n}{n^{k}} \mathbb{E}\left(\left(X_{n}-1\right)^{k}\right) \rightarrow \frac{1}{k}
$$

for $n \rightarrow \infty$. The theorem is now proven as

$$
\frac{\log n}{n^{k}} \mathbb{E}\left(X_{n}^{k}\right)=\sum_{i \in[k]_{0}}\left(\begin{array}{l}
k \\
i
\end{array}\right) \frac{\log n}{n^{k}} \mathbb{E}\left(\left(X_{n}-1\right)^{i}\right) \rightarrow \frac{1}{k}
$$

for $n \rightarrow \infty$.

Remark 3. This last result fits well with the notion that $X_{n}$ can heuristically be seen as $n^{U}$ when $n$ is large ( $U$ uniformly distributed on $[0,1]$ ) following from Theorem 1 , since the $k$ th moment of $n^{U}$ is $n^{k} / k \log n$.

Let us compare this heuristic to the results for $X_{n}$ in Kingman's $n$-coalescent from [4, p. 4], where the authors stated that $X_{n}$, without scaling, converges to a Yule distribution of parameter $\rho=2$. In the Bolthausen-Sznitman $n$-coalescent, the minimal clade size is much bigger than in Kingman's coalescent. This agrees with the more starlike shape of a non-Kingman $n$-coalescent compared to Kingman's $n$-coalescent.

\section{Acknowledgements}

We thank an anonymous referee for pointing out the connection between the minimal clade and the nonrelatives of 1 in the last coalescence event of the Bolthausen-Sznitman $n$-coalescent, and, hence, for improving the proof of the convergence part of Theorem 1. We thank J.-S. Dhersin, M. Möhle, and L. Yuan for a fruitful discussion concerning the alternative proof of Theorem 1, and also E. Teufl for advice concerning which journal to submit to.

\section{References}

[1] Arratia, R., Barbour, A. D. And Tavaré, S. (2003). Logarithmic Combinatorial Structures: A Probabilistic Approach. European Mathematical Society, Zürich.

[2] Berestycki, J., Berestycki, N. ANd Schweinsberg, J. (2013). The genealogy of branching Brownian motion with absorption. Ann. Prob. 41, 527-618.

[3] Bingham, N. H., Goldie, C. M. and Teugels, J. L. (1987). Regular Variation (Encyclopedia Math. Appl. 27). Cambridge University Press.

[4] Blum, M. G. B. And François, O. (2005). Minimal clade size and external branch length under the neutral coalescent. Adv. Appl. Prob. 37, 647-662.

[5] Bolthausen, E. and Sznitman, A.-S. (1998). On Ruelle's probability cascades and an abstract cavity method. Commun. Math. Phys. 197, 247-276.

[6] Bovier, A. And Kurkova, I. (2007). Much ado about Derrida's GREM. In Spin Glasses (Lecture Notes Math. 1900), Springer, Berlin, pp. 81-115.

[7] Brunet, É. And Derrida, B. (2013). Genealogies in simple models of evolution. J. Statist. Mech. Theory Exp. 2013, P01006.

[8] Brunet, É., Derrida, B., Mueller, A. H. And Munier, S. (2006). Noisy traveling waves: effect of selection on genealogies. Europhys. Lett. 76, 1-7.

[9] Brunet, É., Derrida, B., Mueller, A. H. and Munier, S. (2007). Effect of selection on ancestry: an exactly soluble case and its phenomenological generalization. Phys. Rev. E 76, 041104.

[10] Caliebe, A., Neininger, R., Krawczak, M. and Rösler, U. (2007). On the length distribution of external branches in coalescence trees: genetic diversity within species. Theoret. Pop. Biol. 72, 245-252.

[11] Delaurentis, J. M. and Pittel, B. G. (1985). Random permutations and Brownian motion. Pacific J. Math. 119, 287-301.

[12] Desai, M. M., WalczaK, A. M. And Fisher, D. S. (2013). Genetic diversity and the structure of genealogies in rapidly adapting populations. Genetics $193,565-585$. 
[13] Dhersin, J.-S., Freund, F., Siri-JÉgousse, A. And Yuan, L. (2013). On the length of an external branch in the beta-coalescent. Stoch. Process. Appl 123, 1691-1715.

[14] Freund, F. And Möhle, M. (2009). On the time back to the most recent common ancestor and the external branch length of the Bolthausen-Sznitman coalescent. Markov Process. Relat. Fields 15, 387-416.

[15] Fu, Y. X. AND LI, W. H. (1993). Statistical tests of neutrality of mutations. Genetics 133, 693-709.

[16] Gnedin, A., Iksanov, A. And MöHle, M. (2008). On asymptotics of exchangeable coalescents with multiple collisions. J. Appl. Prob. 45, 1186-1195.

[17] Goldschmidt, C. And Martin, J. B. (2005). Random recursive trees and the Bolthausen-Sznitman coalescent. Electron. J. Prob. 10, 718-745.

[18] Hansen, J. C. (1990). A functional central limit theorem for the Ewens sampling formula. J. Appl. Prob. 27, 28-43.

[19] Hwang, H.-K. (1995). Asymptotic expansions for the Stirling numbers of the first kind. J. Combin. Theory A 71, 343-351.

[20] Kingman, J. F. C. (1982). The coalescent. Stoch. Process. Appl. 13, 235-248.

[21] Neher, R. and Hallatschek, O. (2013). Genealogies of rapidly adapting populations. Proc. Nat. Acad. Sci. USA 110, 437-442.

[22] Pitman, J. (1999). Coalescents with multiple collisions. Ann. Prob. 27, 1870-1902.

[23] Pitman, J. (2005). Combinatorial Stochastic Processes (Lecture Notes Math. 1875). Springer, Berlin.

[24] SAgitov, S. (1999). The general coalescent with asynchronous mergers of ancestral lines. J. Appl. Prob. 36, 1116-1125. 